EPJ Web of Conferences 108, 02027 (2016)

DOI: $10.1051 /$ epjconf/201610802027

(C) Owned by the authors, published by EDP Sciences, 2016

\title{
Operator Approach to the Master Equation for the One-Step Process
}

\author{
M. Hnatič ${ }^{3,4,5, a}$, E. G. Eferina ${ }^{1, b}$, A. V. Korolkova ${ }^{1, c}$, D. S. Kulyabov ${ }^{1,2, d}$, and L. A. Sevastyanov ${ }^{1,3, e}$ \\ ${ }^{1}$ Department of Applied Probability and Informatics, Peoples' Friendship University of Russia, \\ Miklukho-Maklaya str. 6, Moscow, 117198, Russia \\ ${ }^{2}$ Laboratory of Information Technologies, Joint Institute for Nuclear Research, \\ Joliot-Curie 6, Dubna, Moscow region, 141980, Russia \\ ${ }^{3}$ Bogoliubov Laboratory of Theoretical Physics, Joint Institute for Nuclear Research, \\ Joliot-Curie 6, Dubna, Moscow region, 141980, Russia \\ ${ }^{4}$ Department of Theoretical Physics, SAS, Institute of Experimental Physics, Watsonova 47, 04001 Košice, \\ Slovakia \\ ${ }^{5}$ Faculty of Science, P. J. Šafárik University, Šrobárova 2, 04154 Košice, Slovakia
}

\begin{abstract}
Background. Presentation of the probability as an intrinsic property of the nature leads researchers to switch from deterministic to stochastic description of the phenomena. The kinetics of the interaction has recently attracted attention because it often occurs in the physical, chemical, technical, biological, environmental, economic, and sociological systems. However, there are no general methods for the direct study of this equation. The expansion of the equation in a formal Taylor series (the so called Kramers-Moyal's expansion) is used in the procedure of stochastization of one-step processes.

Purpose. However, this does not eliminate the need for the study of the master equation. MетноD. It is proposed to use quantum field perturbation theory for the statistical systems (the so-called Doi method).

REsults. This work is a methodological material that describes the principles of master equation solution based on quantum field perturbation theory methods. The characteristic property of the work is that it is intelligible for non-specialists in quantum field theory. Conclusions. We show the full equivalence of the operator and combinatorial methods of obtaining and study of the one-step process master equation.
\end{abstract}

\section{Introduction}

In order to construct stochastic models of the one-step processes [1] (birth-death processes) the combinatorial methodology based on N. G. van Kampen [2] and C. W. Gardiner [3] ideology was

\footnotetext{
ae-mail: hnatic@saske.sk

be-mail: eg.eferina@gmail.com

ce-mail: akorolkova@sci.pfu.edu.ru

de-mail: yamadharma@gmail.com

e-mail: leonid.sevast@gmail.com
} 
worked out. Under this methodology the master equation (for one-step processes) is derived by using the interaction schemes. The obtained master equation is further converted to the Fokker-Planck equation by expansion in formal series (Kramers-Moyal's decomposition) [3]. However, it is necessary to study the possibility of using this expansion for each type of process.

Thus, it is necessary not only to study the master equation but also to justify its expansion. It seems that the quantum perturbation theory best fits all the requirements.

There are two types of formalism which are generally used in the quantum perturbation theory: the path integral formalism and the formalism of second quantization (canonical formalism). It is worthy of note that it is a matter of taste which type of formalism to use. In a number of works [4-7] the possibility of using the formalism of the second quantization for statistical tasks was studied. However, these articles are intended for theoretical physicist and that strongly limits the audience that could use the scientific results of the articles.

The structure of the article is as follows. Section 2 contains a brief introduction to the method of stochastization of one-step processes. Section 3 describes the algorithm of the one-step processes recording in terms of the occupation number representation. The master equation in the form of the Liouville operator equation is also presented. In the section 4 case study model for both the combinatorial and operator approaches is described. The equivalence of the combinatorial and the operator approaches is proved.

\section{One-step processes stochastization}

The one-step processes (also known as the birth-death processes) are Markov processes with continuous time, integer state of states the transition matrix of which allows only transitions between neighbouring states.

\subsection{Interaction schemes}

The system state is defined by the vector $\varphi^{i} \in \mathbb{R}^{n}$, where $n$ is system order ${ }^{1}$. The operator $I_{j}^{i} \in \mathbb{N}_{0}^{n} \times \mathbb{N}_{0}^{n}$ describes the state of the system before the interaction, the operator $F_{j}^{i} \in \mathbb{N}_{0}^{n} \times \mathbb{N}_{0}^{n}$ is the state after the interaction ${ }^{2}$. The result of the interaction is the system transition from one state to another one.

The interaction of the system elements will be described by interaction schemes which are similar to the schemes of the chemical kinetics:

$$
I_{j}^{i \alpha} \varphi^{j} \underset{-k_{\alpha}}{\stackrel{+}{{ }_{k}}} F_{j}^{i \alpha} \varphi^{j}
$$

the Greek indices specify the number of interactions and the Latin ones the system order. The coefficients ${ }^{+} k_{\alpha}$ and ${ }^{-} k_{\alpha}$ have the meaning of intensity (speed) of the interaction.

The state transition is given by the operator: $r_{j}^{i \alpha}=F_{j}^{i \alpha}-I_{j}^{i \alpha}$. Thus, the one step interaction $\alpha$ in the forward and reverse directions can be written as $\varphi^{i} \rightarrow \varphi^{i}+r_{j}^{-i \alpha} \varphi^{j}, \quad \varphi^{i} \rightarrow \varphi^{i}-r_{j}^{i \alpha} \varphi^{j}$.

We can also write (1) not as vector equations but as sums: $I_{j}^{i \alpha} \varphi^{j} \delta_{i} \underset{-{ }_{k_{\alpha}}}{\stackrel{+}{+} k_{\alpha}} F_{j}^{i \alpha} \varphi^{j} \delta_{i}$, where $\delta_{\underline{i}}=(1, \ldots, 1)$. Also the following notation will be used: $I^{i \alpha}:=I_{j}^{i \alpha} \delta^{j}, F^{i \alpha}:=F_{j}^{i \alpha} \delta^{j}, r^{i \alpha}:=r_{j}^{i \alpha} \delta^{j}$.

\footnotetext{
${ }^{1}$ For brevity, we denote the module over the field $\mathbb{R}$ just as $\mathbb{R}$.

${ }^{2}$ The component dimension indices take values $\underline{i}, \underline{j}=\overline{1, n}$.
} 


\subsection{The master equation}

For the system description we will use the master equation, which describes the transition probability for a Markov process $[2,3]$. If the domain of variation of $\varphi$ is discrete, then the master equation can be written as follows (the states are numbered by $n$ and $m$ ):

$$
\frac{\partial p_{n}(t)}{\partial t}=\sum_{m}\left[w_{n m} p_{m}(t)-w_{m n} p_{n}(t)\right]
$$

where $p_{n}$ is the probability to find the system in the state $n$ at the time $t, w_{n m}$ is the probability of the transition from the state $m$ to the state $n$ per unit time.

There are two types of system transitions from one state to another (based on one-step processes) as a result of system elements interaction: in the forward direction $\left(\varphi^{i}+r_{j}^{-i \alpha} \varphi^{j}\right)$ with probability ${ }^{+} s_{\underline{\alpha}}\left(\varphi^{k}\right)$ and in the opposite direction $\left(\varphi^{i}-r_{j}^{i \alpha} \varphi^{j}\right)$ with probability ${ }^{-} s_{\alpha}\left(\varphi^{k}\right)$. The matrix of the transition probabilities has the form: $w_{\underline{\alpha}}\left(\varphi^{i} \mid \psi^{i}, t\right)={ }^{+} s_{\alpha} \delta_{\varphi^{i}, \psi^{i}+1}+{ }^{-} s_{\alpha} \delta_{\varphi^{i}, \psi^{i}-1}$, where $\delta_{i, j}$ is Kronecker delta.

Thus, the general form of the master equation for the state vector $\varphi^{i}$, changing by steps of length $r_{j}^{i \alpha} \varphi^{j}$, is:

$$
\begin{aligned}
\frac{\partial p\left(\varphi^{i}, t\right)}{\partial t}=\sum_{\underline{\alpha}=1}^{s}\left\{\left[-{ }_{\underline{\alpha}}\left(\varphi^{i}+r_{-}^{i \alpha}, t\right) p\left(\varphi^{i}+r_{-}^{i \alpha}, t\right)\right.\right. & \left.-{ }^{+} s_{\underline{\alpha}}\left(\varphi^{i}\right) p\left(\varphi^{i}, t\right)\right]+ \\
& \left.+\left[{ }^{+} s_{\underline{\alpha}}\left(\varphi^{i}-r^{i \alpha}, t\right) p\left(\varphi^{i}-r^{i \alpha}, t\right)-{ }^{+} s_{\underline{\alpha}}\left(\varphi^{i}\right) p\left(\varphi^{i}, t\right)\right]\right\} .
\end{aligned}
$$

The transition rates ${ }^{+} s_{\alpha}$ and ${ }^{-} s_{\alpha}$ are proportional to the number of ways of choosing the number of arrangements of $\varphi_{-}^{i}$ to $I_{-}^{i \alpha}$ (denoted as $\left.A_{\varphi_{-}^{i}}^{I \frac{i \alpha}{i}}\right)$ and to $F^{i \alpha}$ (denoted as $\left.A_{\varphi_{-}^{-}}^{F_{i}^{i \alpha}}\right)$ and defined by:

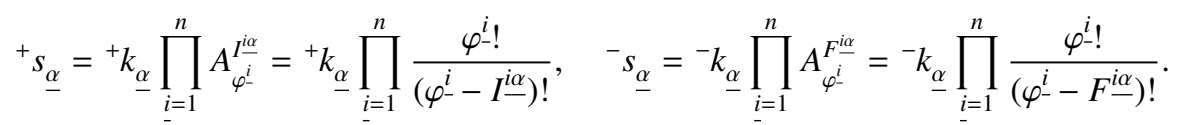

Replacing in (4) the $\varphi(\varphi-1) \cdots(\varphi-(n-1))$-type combinations on $(\varphi)^{n}$ we obtain for Fokker-Planck equation ${ }^{3}$ :

$$
{ }_{\mathrm{fp}}^{+} s_{\underline{\alpha}}={ }^{+} k_{\underline{\alpha}} \prod_{\underline{i}=1}^{n}\left(\varphi^{i}\right)^{i}{ }^{i \underline{\underline{\alpha}}}, \quad \mathrm{fp}_{\underline{-}}^{-} s_{\alpha}={ }^{-} k_{\underline{\alpha}} \prod_{\underline{i}=1}^{n}\left(\varphi^{i}\right)^{F^{i \underline{\alpha}}} .
$$

\section{Occupation numbers representation}

The occupation number representation is the main language in the description of many-body physics. The main elements of the language are the wave functions of the system, providing information about how many particles are in each single-particle state. The creation and annihilation operators are used for system states change. The advantages of this formalism are:

- it is possible to consider systems with a variable number of particles (non-stationary systems);

- the system statistics (Fermi-Dirac or Bose-Einstein) is automatically included in the commutation rules for the creation and annihilation operators;

\footnotetext{
${ }^{3}$ This change corresponds to a series expansion.
} 
- this is the second major formalism (along with the path integral) for the quantum perturbation theory description.

The application of the formalism of the second quantization to non-quantum systems (statistical, deterministic systems) was also studied [4, 8-10].

The Dirac notation is commonly used for occupation numbers representation recording.

\subsection{Dirac notation}

The vector $\varphi^{i}$ is denoted $|i\rangle$, the covariant vector (covector) $\varphi_{i}$ is denoted $\langle i|$. The conjunction operation is used for raising and lowering the indices ${ }^{4}: \varphi_{i}^{*}:=\varphi_{i}=\left(\varphi^{i}\right)^{\dagger} \equiv\langle i|=| i\rangle^{\dagger}$. The scalar product is: $\varphi_{i} \varphi^{i} \equiv\langle i \mid i\rangle$. The tensor product is: $\varphi_{j} \varphi^{i} \equiv|i\rangle\langle j|$. Another form of the Dirac notation is also possible: $|\varphi\rangle:=\varphi^{i}, \quad\langle i \mid \varphi\rangle:=\varphi^{i} \delta_{i}^{i}=\varphi_{-}^{i}$

\subsection{Creation and annihilation operators}

The transition to the space of occupation numbers is not a unitary transformation. However, the algorithm of the transition (specific to each task) can be constructed.

This procedure is illustrated here for the master equation (2) for a system which does not depend on the spatial variables and is one-dimensional.

The probability that the system of interest consists of $n$ particles is

$$
\varphi_{n}:=p_{n}(\varphi, t)
$$

The vector space $\mathcal{H}$ consists of states of $\varphi$. The scalar product can be written:

$$
\langle\varphi \mid \psi\rangle=\sum_{n} n ! p_{n}^{*}(\varphi) p^{n}(\psi)=\sum_{n} n ! \varphi_{n}^{*}(\varphi) \varphi^{n}(\psi)
$$

From $p_{n}(m)=\delta_{n}^{m}$ and (7) we obtain:

$$
\langle n \mid m\rangle=n ! \delta_{n}^{m}
$$

The state vector:

$$
|\varphi\rangle=\sum_{n} p_{n}(\varphi)|n\rangle=\sum_{n} \varphi_{n}|n\rangle=: \varphi_{n}|n\rangle
$$

In view of (8), we get:

$$
\varphi_{n}=\frac{1}{n !}\langle n \mid \varphi\rangle
$$

Creation and annihilation operators are defined respectively as:

$$
\pi|n\rangle=|n+1\rangle, \quad a|n\rangle=n|n-1\rangle .
$$

They satisfy the commutation rule ${ }^{5}$ :

$$
[a, \pi]=1 .
$$

From (7) and (12) it follows that the system is described by Bose-Einstein statistics. From (8) we get: $\left\langle m\left|a^{\dagger}\right| n\right\rangle=\langle m|\pi| n\rangle$, and therefore: $a^{\dagger}=\pi$.

\footnotetext{
${ }^{4}$ In this case, we use Hermitian conjugation $\bullet{ }^{\dagger}$. The sign of the complex conjugate $\bullet *$ in this entry is superfluous.

${ }^{5}$ In fact, $a \pi|n\rangle-\pi a|n\rangle=(n+1)|n\rangle-n|n\rangle=|n\rangle$.
} 


\subsection{Liouville operator}

The Liouville equation:

$$
\frac{\partial}{\partial t}|\varphi(t)\rangle=L|\varphi(t)\rangle
$$

Liouville operator $L$ satisfies the relation: $\langle 0| L=0$.

From (2), (9), (10) and (13) we obtain:

$$
\frac{\partial p_{n}}{\partial t}=\frac{1}{n !}\left\langle n\left|\frac{\partial}{\partial t}\right| \varphi\right\rangle=\frac{1}{n !}\langle n|L| \varphi\rangle .
$$

In this way, the system of master equations (2) has been reduced to a single equation, the Liouville equation (13). The following Liouville operator corresponds to the scheme (1):

$$
L=\sum_{\underline{\alpha}, \underline{\underline{L}}}\left[{ }^{+} k_{\underline{\alpha}}\left(\left(\pi_{\underline{i}}\right)^{F^{i \underline{\alpha}}}-\left(\pi_{i}\right)^{I^{i \underline{\alpha}}}\right)\left(a_{\underline{\underline{ }}}\right)^{I^{i \underline{\alpha}}}+{ }^{-} k_{\underline{\alpha}}\left(\left(\pi_{\underline{i}}\right)^{I^{i \underline{\alpha}}}-\left(\pi_{\underline{i}}\right)^{F^{i \underline{\alpha}}}\right)\left(a_{\underline{i}}\right)^{F^{i \underline{\alpha}}}\right] .
$$

\section{Verhulst model}

As a demonstration of the method, we consider the Verhulst model [11], which describes the limited growth $^{6}$. Initially, this model was written down as the differential equation: $\frac{\mathrm{d} \varphi}{\mathrm{d} t}=\lambda \varphi-\beta \varphi-\gamma \varphi^{2}$, where $\lambda$ denotes the breeding intensity factor, $\beta$ - the extinction intensity factor, $\gamma$ - the factor of population reduction rate (usually the rivalry of individuals is considered) ${ }^{7}$.

The interaction scheme for the stochastic version of the model is:

$$
\varphi \stackrel{\lambda}{\underset{\gamma}{\rightleftharpoons}} 2 \varphi, \quad \varphi \stackrel{\beta}{\rightarrow} 0 . \quad I^{i \alpha}=\left(\begin{array}{ll}
1 & 1
\end{array}\right), \quad F^{i \alpha}=\left(\begin{array}{ll}
2 & 0
\end{array}\right) . \quad r^{i \alpha}=\left(\begin{array}{ll}
1 & -1
\end{array}\right) .
$$

The first relation means that an individual who eats one unit of meal is immediately reproduced, and in the opposite direction is the rivalry between individuals. The second relation describes the death of an individual.

\subsection{One-step processes stochastization method}

The correspondence between the terms entering the Fokker-Plank equation (5) and the transition rates defined within the Verhults model is as follows: ${ }^{+} s_{1}=\lambda \varphi,{ }^{-} s_{1}=\gamma \varphi(\varphi-1),{ }^{+} s_{2}=\beta \varphi$; ${ }_{\mathrm{fp}}^{+} s_{1}=\lambda \varphi, \quad{ }_{\mathrm{fp}} s_{1}=\gamma \varphi^{2}, \quad{ }_{\mathrm{fp}}^{+} s_{2}=\beta \varphi$. Then, based on (3), the form of the master equation is:

$$
\frac{\partial p(\varphi, t)}{\partial t}=-[\lambda \varphi+\beta \varphi+\gamma \varphi(\varphi-1)] p(\varphi, t)+[\beta(\varphi+1)+\gamma(\varphi+1) \varphi] p(\varphi+1, t)+\lambda(\varphi-1) p(\varphi-1, t) .
$$

For particular values of $\varphi($ as in (2)):

$$
\frac{\partial p_{n}(t)}{\partial t}:=\left.\frac{\partial p(\varphi, t)}{\partial t}\right|_{\varphi=n}=-[\lambda n+\beta n+\gamma n(n-1)] p_{n}(t)+[\beta(n+1)+\gamma(n+1) n] p_{n+1}(t)+\lambda(n-1) p_{n-1}(t) .
$$

\footnotetext{
${ }^{6}$ The attractiveness of this model is that it is one-dimensional and non-linear.

${ }^{7}$ The same notation as in the original model [11] is used.
} 


\subsection{Occupation number representation}

From (16) and (15) the Liouville operator is:

$$
L=\lambda\left(\pi^{2}-\pi\right) a+\gamma\left(\pi-\pi^{2}\right) a^{2}+\beta(1-\pi) a=\lambda\left(a^{\dagger}-1\right) a^{\dagger} a+\beta\left(1-a^{\dagger}\right) a+\gamma\left(1-a^{\dagger}\right) a^{\dagger} a^{2} .
$$

The master equation by Liouville operator (from (14)) and by means of (8), (11), (6) and (10):

$$
\begin{aligned}
\frac{\partial p_{n}(t)}{\partial t}=\frac{1}{n !}\langle n|L| \varphi\rangle=\frac{1}{n !}\left\langle n\left|-\left[\lambda a^{\dagger} a+\beta a^{\dagger} a+\gamma a^{\dagger} a^{\dagger} a a\right]+\left[\beta a+\gamma a^{\dagger} a a\right]+\lambda a^{\dagger} a^{\dagger} a\right| \varphi\right\rangle= \\
=-[\lambda n+\beta n+\gamma n(n-1)] p_{n}(t)+[\beta(n+1)+\gamma(n+1) n] p_{n+1}(t)+\lambda(n-1) p_{n-1}(t) .
\end{aligned}
$$

The result (18) coincides with (17), which was obtained by the combinatorial method.

\section{Conclusions}

This article introduced the operator method for one-step processes. At all stages of the operator method it is compared with the combinatorial method of stochastization of the one-step processes. The logic of both methods is demonstrated. The complete equivalence of both methods is presented by their comparison.

However, at this stage it is a difficult task to justify a preference for one of the methods. But it should be noted that the operator formalism allows to use the achievements made within the framework of the quantum field theory in a more familiar way.

\section{Acknowledgements}

The work was partially supported by RFBR grants No's 14-01-00628 and 15-07-08795. This work was supported by Vega grant 1/0222/13.

\section{References}

[1] E.G. Eferina, A.V. Korolkova, M.N. Gevorkyan, D.S. Kulyabov, L.A. Sevastyanov, Bulletin of Peoples' Friendship University of Russia. Series "Mathematics. Information Sciences. Physics" pp. 46-59 (2014), 1503.07342

[2] N.G. van Kampen, Stochastic Processes in Physics and Chemistry, North-Holland Personal Library (Elsevier Science, 2011), ISBN 9780080475363

[3] C.W. Gardiner, Handbook of Stochastic Methods: for Physics, Chemistry and the Natural Sciences (Springer Series in Synergetics, 1985)

[4] M. Doi, Journal of Physics A: Mathematical and General 9, 1465 (1976)

[5] M. Hnatich, J. Honkonen, Physical review. E, Statistical physics, plasmas, fluids, and related interdisciplinary topics 61, 3904 (2000)

[6] M. Hnatich, J. Honkonen, T. Lučivjanský, Theoretical and Mathematical Physics 169, 1489 (2011), 1109.6435

[7] M. Hnatič, J. Honkonen, T. Lučivjanský, Physics of Particles and Nuclei 44, 316 (2013)

[8] Y.B. Zel'dovich, A.A. Ovchinnikov, Sov. Phys. JETP 47, 829 (1978)

[9] P. Grassberger, M. Scheunert, Fortschritte der Physik 28, 547 (1980)

[10] L. Peliti, Journal de Physique 46, 1469 (1985)

[11] P.F. Verhulst, Notice sur la loi que la population suit dans son accroissement, Vol. 10 (1838) 